OPEN ACCESS

Edited by:

Pavel Lindberg,

INSERM U894 Centre de Psychiatrie

et Neurosciences, France

Reviewed by:

Isabelle Loubinoux

INSERM U1214 Centre d'Imagerie

Neuro Toulouse (ToNIC), France

Assia Jaillard,

Université Grenoble Alpes, France

${ }^{*}$ Correspondence:

Leeanne M. Carey

I.carey@/atrobe.edu.au

Specialty section:

This article was submitted to

Stroke,

a section of the journal

Frontiers in Neurology

Received: 13 September 2018

Accepted: 10 December 2018

Published: 10 January 2019

Citation:

Lamp G, Goodin P, Palmer S, Low E,

Barutchu A and Carey LM (2019)

Activation of Bilateral Secondary

Somatosensory Cortex With Right

Hand Touch Stimulation: A

Meta-Analysis of Functional

Neuroimaging Studies.

Front. Neurol. 9:1129.

doi: 10.3389/fneur.2018.01129

\section{Activation of Bilateral Secondary Somatosensory Cortex With Right Hand Touch Stimulation: A Meta-Analysis of Functional Neuroimaging Studies}

\author{
Gemma Lamp 1,2, Peter Goodin ${ }^{1}$, Susan Palmer ${ }^{1}$, Essie Low ${ }^{1,3,4}$, Ayla Barutchu ${ }^{1,5}$ and \\ Leeanne M. Carey ${ }^{1,2 *}$ \\ ${ }^{1}$ Neurorehabilitation and Recovery, Florey Institute of Neuroscience and Mental Health, Melbourne Brain Centre, Heidelberg, \\ VIC, Australia, ${ }^{2}$ Occupational Therapy, School of Allied Health, La Trobe University, Bundoora, VIC, Australia, ${ }^{3}$ Department of \\ Neurology, Sunshine Hospital, Western Health, Melbourne, VIC, Australia, ${ }^{4}$ Department of Psychology, Royal Adelaide \\ Hospital, Adelaide, SA, Australia, ${ }^{5}$ Balliol College, University of Oxford, Oxford, United Kingdom
}

Background: Brain regions involved in processing somatosensory information have been well documented through lesion, post-mortem, animal, and more recently, structural and functional neuroimaging studies. Functional neuroimaging studies characterize brain activation related to somatosensory processing; yet a meta-analysis synthesis of these findings is currently lacking and in-depth knowledge of the regions involved in somatosensory-related tasks may also be confounded by motor influences.

Objectives: Our Activation Likelihood Estimate (ALE) meta-analysis sought to quantify brain regions that are involved in the tactile processing of the right $(\mathrm{RH})$ and left hands $(\mathrm{LH})$ separately, with the exclusion of motor related activity.

Methods: The majority of studies $(n=41)$ measured activation associated with $\mathrm{RH}$ tactile stimulation. $\mathrm{RH}$ activation studies were grouped into those which conducted whole-brain analyses $(n=29)$ and those which examined specific regions of interest (ROI; $n=12)$. Few studies examined LH activation, though all were whole-brain studies $(N=7)$.

Results: Meta-analysis of brain activation associated with $\mathrm{RH}$ tactile stimulation (whole-brain studies) revealed large clusters of activation in the left primary somatosensory cortex (S1) and bilaterally in the secondary somatosensory cortex (S2; including parietal operculum) and supramarginal gyrus (SMG), as well as the left anterior cingulate. Comparison between findings from $\mathrm{RH}$ whole-brain and $\mathrm{ROI}$ studies revealed activation as expected, but restricted primarily to $\mathrm{S} 1$ and $\mathrm{S} 2$ regions. Further, preliminary analyses of LH stimulation studies only, revealed two small clusters within the right S1 and S2 regions, likely limited due to the small number of studies. Contrast analyses revealed the one area of overlap for $\mathrm{RH}$ and $\mathrm{LH}$, was right secondary somatosensory region.

Conclusions: Findings from the whole-brain meta-analysis of right hand tactile stimulation emphasize the importance of taking into consideration bilateral activation, particularly in secondary somatosensory cortex. Further, the right parietal operculum/S2 
region was commonly activated for right and left hand tactile stimulation, suggesting a lateralized pattern of somatosensory activation in right secondary somatosensory region. Implications for further research and for possible differences in right and left hemispheric stroke lesions are discussed.

Keywords: ALE "activation likelihood estimation", meta-analysis, brain activation, sensation, hand, touch, secondary somatosensory cortex

\section{INTRODUCTION}

Somatosensory function is crucial for daily life, guiding our interactions with the world around us through the detection, discrimination and recognition of body sensations (1). Somatosensation is important not only for perception, but also for goal-directed action (2, 3). For example, somatosensation contributes to the fundamental pinch grip-lift-and hold task (4) and is important in dexterous movement of the hand (5). Following stroke, reduced functional arm use is contributed to by motor and somatosensory deficits. Somatosensory impairment has a negative impact on grasp and manipulation of objects (6) and is associated with reduced arm use (7). Further, somatosensory brain regions have been implicated in motor recovery (8). It has been suggested that somatosensory processing for the guidance of action can be dissociated from the processing that leads to perception (2). Here we focus on brain regions involved in somatosensation, specifically tactile stimulation of the hand, without motor confounds.

The neuroanatomy of somatosensory processing is well established through a large body of lesion, post-mortem, animal and structural neuroimaging studies (9-12). Reproducible functional activation in the contralateral primary somatosensory cortex (S1) has been demonstrated in healthy controls when asked to perceive a touch stimulus to their fingertips (13). Technological advances in recent years have even allowed mapping of individual fingers to corresponding areas of S1 (14) and the temporal acuity of anticipation of a tactile stimulus originating in the ipsilateral S1 (15).

Different patterns of activation and lateralization emerge when examining somatosensory processing in the secondary somatosensory cortex (S2). Median nerve stimulation has been shown to activate bilateral S2 regions, including parietal operculum, regardless of the hand being stimulated, but only the contralateral S1 (16). This has also been seen in other stimulation studies. Lee et al. (17) recently examined the differential neural activations associated with vibrotactile, pressure and temperature stimulation of right palm, showing common activation in the contralateral S1 and bilateral S2/insula regardless of stimulation type. Bilateral S2 region activation has also been seen with vibrotactile stimulation irrespective of other cognitive demands (18). It has been suggested that serial somatosensory processing occurs from contralateral S1 to contralateral S2 in response to electrical stimulation, but when stimulation becomes more intense or painful there is an increase in hemispheric integration (19). A meta-analysis of studies examining the functional role of S2 in somatosensory processing divided the area into OP1 (parietal operculum 1), OP2, OP3, and OP4 (10). While OP1 is reported to represent the human homolog of macaque area S2 and was generally more responsive to pure somatosensory (tactile) stimuli (10), overall the areas were all implicated in different somatosensory processes (20). A thorough review of the functional role of S2, from the bi-laterality of activation with unilateral stimulation, to the mapping of the hand area spread of OP1-OP4, has been provided by Eickhoff et al. (10).

When examining the literature it becomes clear that the functional activation of somatosensory processing in the brain is still a developing area. There are various stimulation techniques to investigate reflexive neural activity, for example vibrotactile stimulation $(18,21)$ as opposed to MNS median nerve stimulation $(16,22,23)$, that can yield different results. Somatosensory stimuli are applied to various body parts, including the face, upper limb, and lower limb (10, 24), but may not be performed on each hand separately $(25,26)$. Finally, studies have often been confounded by motor contributions to the task, e.g., involving movement intention and/or execution (27-29).

Our aim was to characterize and synthesize the somatosensory brain activation network during touch sensation, with potential influence of motor contributions eliminated. We employed the ALE meta-analytic technique to provide a statistically-based likelihood estimation of the brain regions that are consistently activated during tactile stimulation of the hands. Firstly, studies were limited to those that involved only tactile stimulation of the right $(\mathrm{RH})$ or left hand $(\mathrm{LH})$ separately in order to allow interpretation of networks that account for hemispheric dominance. Following this, studies which incorporated any motor movements during the stimulation task were excluded, to address confounding motor influence during somatosensory task performance. Lastly, to characterize neural correlates specific to touch sensation, studies involving other somatosensory modalities, such as pain or proprioception, were excluded.

\section{METHODS}

\section{Identification of Studies for Meta-Analysis}

The meta-analysis of neuroimaging studies was conducted according to the PRISMA statement and recorded using the suggested checklist (30). A thorough literature search was conducted using Web of Science database (conducted December 12, 2017) and the following search terms: (fMRI OR MRI OR PET OR "functional magnetic resonance imaging" OR "positron emission tomography" OR neuroimaging OR "brain imaging" OR "neural activation") AND (somatosen* OR sens* OR tactile) AND (hand OR "upper limb" OR finger) AND (health* OR control). These papers were then crosschecked with 
papers identified in the Sleuth functional database (31-33). The Sleuth database was searched for "somesthesis perception" in the behavioral domain and for "activation only" studies. These were reviewed using the strict inclusion criteria (see Figure 1).

\section{Activation Likelihood Estimation Meta-Analysis}

The meta-analysis was performed using Activation Likelihood Estimation (ALE) on the activation voxel coordinates reported by the selected study (34-36). Analyses were conducted using GingerALE (version 2.3.6) (37) software (downloaded from http://brainmap.org/ale), with coordinates in Montreal Neurological Institute (MNI) space (38, 39). Coordinates reported in Talairach space (40) were converted to MNI space using the "icbm2tal transform" $(41,42)$. To minimize withinexperiment and within-group effects we utilized the modified algorithm described in Turkeltaub, Eickhoff (36) and, thus, were able to include multiple contrasts from within the one study. The calculated ALE map had a cluster forming threshold of $p<0.001$ with 1000 permutations, corrected for multiple comparisons using the Family Wise Error (FWE) $p<0.05$ $(20,37,43,44)$. Contrast and conjunction analyses were calculated to compare activation associated with task type, first by creating an image of two tasks pooled together (e.g., $\mathrm{RH}$ and $\mathrm{LH}$ ) with an uncorrected threshold of $p<0.01$ at 10,000 permutations, and then subtracting each original task analysis




TABLE 1 | Studies included in the meta-analysis $(n=45)$ and reported participant and task information, separated by task category.

\begin{tabular}{|c|c|c|c|c|c|c|c|c|c|}
\hline References & $N$ & $\begin{array}{l}\text { Age } \\
\text { M (SD); } \\
\text { min-max }\end{array}$ & $\begin{array}{l}\text { Sex } \\
\text { M:F }\end{array}$ & Handedness & Stimulus type & $\begin{array}{l}\text { Stimulus } \\
\text { Location }\end{array}$ & Attended & $\begin{array}{l}\text { Response } \\
\text { required }\end{array}$ & fMRI/PET \\
\hline \multicolumn{10}{|c|}{ Right Hand stimulation Whole-Brain $(N=29)$} \\
\hline Borstad et al. (52) & 10 & $39-82$ & $5: 5$ & $9 \mathrm{RH}, 1 \mathrm{LH}$ & Brush stroke & Index finger & Y & $N$ & fMRI \\
\hline Bjornsdotter et al. (53) & 22 & 19-35 & $13: 9$ & NR & Brush stroke & Palm & Y & $\mathrm{N}$ & fMRI \\
\hline Brodoehl et al. (54) & 34 & $21-71$ & $17: 17$ & $\mathrm{RH}$ & Compressed air & Fingers 1-3 & Y & $N$ & $\mathrm{fMRI}$ \\
\hline Brodoehl et al. (55) & 10 & $23.1(1.54)$ & $0: 10$ & $\mathrm{RH}$ & Compressed ait & Fingers 1-5 & Y & $N$ & $\mathrm{fMRI}$ \\
\hline Brodoehl et al. (56) & 32 & $21-71$ & $15: 17$ & $\mathrm{RH}$ & Compressed air & Fingers 1-5 & Y & $N$ & $\mathrm{fMRI}$ \\
\hline Burton et al. (57) & 11 & $19-25$ & $5: 6$ & $\mathrm{RH}$ & Textured surface & Digits 2-3 & Y & Y (after scan) & fMRI \\
\hline Carey et al. $(13)^{\star}$ & 5 & $52-76$ & $3: 2$ & $\mathrm{RH}$ & Texture grids & Fingertips & Y & Y (after scan) & PET \\
\hline Chung et al. (58) & 21 & $24.19(2.71)$ & $N R$ & $\mathrm{RH}$ & Band pressure & Index finger & Y & $N$ & $\mathrm{fMRI}$ \\
\hline Chung et al. (59) & 21 & $24.19(2.17)$ & $N R$ & $\mathrm{RH}$ & Band pressure & Index finger & Y & $\mathrm{N}$ & $\mathrm{fMRI}$ \\
\hline Gelnar et al. (27) & 9 & 18-NR & NR & $\mathrm{RH}$ & Vibration & Fingers 2-5 & Y & $\mathrm{N}$ & fMRI \\
\hline Godde et al. (60) & 10 & $18-30$ & $8: 2$ & $\mathrm{RH}$ & Vibration & Fingers & Y & $N$ & $\mathrm{fMRI}$ \\
\hline Hagen et al. (61)* & 18 & $37(12)$ & $11: 7$ & $\mathrm{RH}$ & Von Frey & Index finger & Y & $N$ & PET \\
\hline $\begin{array}{l}\text { Hlushchuk and Hari, } \\
\text { (62) }\end{array}$ & 10 & $23-33$ & $7: 3$ & NR & Compressed air & $\begin{array}{l}\text { Index, middle, } \\
\text { ring fingers }\end{array}$ & Y & $\mathrm{N}$ & $\mathrm{fMRI}$ \\
\hline $\begin{array}{l}\text { Kavounoudias et al. } \\
\text { (63) }\end{array}$ & 10 & $31.4(10.7)$ & $2: 8$ & $\mathrm{RH}$ & Textured surface & Whole hand & Y & $\mathrm{N}$ & $\mathrm{fMRI}$ \\
\hline Kitada et al. (64) & 5 & $23-25$ & $5: 0$ & $\mathrm{RH}$ & Pressure & First 2 fingers & Y & Y & $\mathrm{fMRI}$ \\
\hline Kitada et al. (65) & 14 & $23-26$ & $12: 2$ & $\mathrm{RH}$ & Tactile grids & 2 Fingers & Y & $N$ & $\mathrm{fMRI}$ \\
\hline Kwon et al. (66) & 10 & $\begin{array}{l}25.20(2.49) \\
22-29\end{array}$ & $5: 5$ & $\mathrm{RH}$ & Rubber brush & Dorsum & Y & $\mathrm{N}$ & $\mathrm{fMRI}$ \\
\hline Lee et al. (17) & 10 & $\begin{array}{l}27.8(4.1) \\
23-34\end{array}$ & $8: 2$ & NR & Vibratory brush & $\begin{array}{l}\text { Palm of right } \\
\text { hand }\end{array}$ & $\mathrm{y}$ & $\mathrm{N}$ & $\mathrm{fMRI}$ \\
\hline Malinen et al. (67) & 10 & 20-32 & $6: 4$ & $\mathrm{RH}$ & Vibration & Fingers 2-3 & NR & NR & fMRI \\
\hline McGlone et al. (68) & 10 & $18-26$ & $0: 10$ & $\mathrm{RH}$ & Brush stroke & Palm & $N R$ & $N R$ & PET \\
\hline Nebel et al. (69) & 12 & $28.7(7.6)$ & $0: 12$ & NA & Vibration & Hand & $N R$ & $N$ & $\mathrm{fMRI}$ \\
\hline Ozcan et al. (70)* & 12 & $22-35$ & $8: 4$ & $11 \mathrm{RH}, 1 \mathrm{LH}$ & Compressed air & Fingertips & $N$ & $\mathrm{~N}$ & fMRI \\
\hline $\begin{array}{l}\text { Planetta and Servos, } \\
\text { (71) }\end{array}$ & 10 & $25(1)$ & $3: 7$ & $\mathrm{RH}$ & Pressure & Fingertips & NR & NR & $\mathrm{fMRI}$ \\
\hline Rolls et al. (72) & 9 & $28(N R)$ & $5: 4$ & $\mathrm{RH}$ & Textured surface & Hand & NR & NR & $\mathrm{fMRI}$ \\
\hline Ruben et al. (73) & 8 & $21-31$ & $6: 2$ & $N R$ & $\begin{array}{l}\text { Electrical } \\
\text { stimulation }\end{array}$ & Digit 2 and 5 & NR & NR & $\mathrm{fMRI}$ \\
\hline Schurmann et al. (74) & 13 & $22-39$ & $9: 4$ & $\mathrm{RH}$ & $\begin{array}{l}\text { Vibration; } \\
\text { Compressed air }\end{array}$ & Hand; Fingers & Y & $N$ & $\mathrm{fMRI}$ \\
\hline Summers et al. (75) & 6 & $20-33$ & $6: 0$ & $\mathrm{RH}$ & Vibration & Digit 2 & Y & $\mathrm{N}$ & fMRI \\
\hline Yoo et al. (76) & 13 & $21-38$ & $8: 5$ & $\mathrm{RH}$ & Von Frey brush & Index finger & Y & $N$ & $\mathrm{fMRI}$ \\
\hline Young et al. (77) & 10 & $21-32$ & $6: 4$ & $\mathrm{RH}$ & Textured surface & Hand & Y & $\mathrm{N}$ & fMRI \\
\hline \multicolumn{10}{|c|}{ Right Hand stimulation Region of Interest (ROI) studies ( $=12$ ) } \\
\hline Blankenburg et al. (78) & 8 & $25-39$ & $7: 1$ & $\mathrm{RH}$ & $\begin{array}{l}\text { Electrical } \\
\text { stimulation }\end{array}$ & $\begin{array}{l}\text { Third finger } \\
\text { and palm }\end{array}$ & NR & $N$ & $\mathrm{fMRI}$ \\
\hline Blatow et al. (79) & 12 & $25-59$ & $5: 7$ & $\mathrm{RH}$ & Vibration & Digits 1 and 2 & Y & Y & fMRI \\
\hline Blatow et al. (80) & 16 & $21-51$ & $8: 8$ & $\mathrm{RH}$ & Vibration & Digits 1 and 2 & $N R$ & $N R$ & $\mathrm{fMRI}$ \\
\hline Burton et al. (81) & 12 & $28.3(12.8)$ & $8: 4$ & $\mathrm{RH}$ & Vibration & Index finger & Y & Y & $\mathrm{fMRI}$ \\
\hline Deuchert et al. (82) & 8 & 23-26 & $4: 4$ & $\mathrm{RH}$ & $\begin{array}{l}\text { Von Frey } \\
\text { monofilaments }\end{array}$ & $\begin{array}{l}\text { Thenar } \\
\text { eminence }\end{array}$ & Y & Y & fMRI \\
\hline Dresel et al. (83) & 6 & 24-39 & $2: 4$ & $5 \mathrm{RH}, 1 \mathrm{LH}$ & $\begin{array}{l}\text { Electrical } \\
\text { stimulation }\end{array}$ & $\begin{array}{l}2 \text { and 5th } \\
\text { finger }\end{array}$ & $\mathrm{N}$ & $\mathrm{N}$ & fMRI \\
\hline Eickhoff et al. (10) & 14 & $25.6(3.4)$ & $7: 7$ & $\mathrm{RH}$ & Brush stroke & Fingers & $\mathrm{Y}$ & Y & fMRI \\
\hline
\end{tabular}


TABLE 1 | Continued

\begin{tabular}{|c|c|c|c|c|c|c|c|c|c|}
\hline References & $N$ & $\begin{array}{l}\text { Age } \\
\text { M (SD); } \\
\text { min-max }\end{array}$ & $\begin{array}{l}\text { Sex } \\
\text { M:F }\end{array}$ & Handedness & Stimulus type & $\begin{array}{l}\text { Stimulus } \\
\text { Location }\end{array}$ & Attended & $\begin{array}{l}\text { Response } \\
\text { required }\end{array}$ & fMRI/PET \\
\hline $\begin{array}{l}\text { Hlushchuk and Hari, } \\
\text { (62) }\end{array}$ & 6 & $20-30$ & $2: 4$ & $\mathrm{RH}$ & Compressed air & Palm & NR & $\mathrm{NR}$ & fMRI \\
\hline Huang and Sereno, (84) & 9 & 23-33 & $6: 3$ & NR & Compressed air & Digits 2,3,4 & Y & $\mathrm{N}$ & $\mathrm{fMRl}$ \\
\hline Kobayashi et al. (85) & 10 & $18-22$ & $0: 10$ & $\mathrm{RH}$ & Textured surface & Palm & Y & $\mathrm{N}$ & fMRI \\
\hline Martuzzi et al. (86) & 10 & 20-35 & $10: 0$ & $\mathrm{RH}$ & Stroke & Finger tips & Y & $\mathrm{N}$ & fMRI \\
\hline Nelson and Chen, (87) & 12 & $25-66$ & $4: 8$ & $\mathrm{RH}$ & Vibration & Fingertip & Y & $\mathrm{N}$ & fMRI \\
\hline \multicolumn{10}{|c|}{ Left Hand stimulation Whole-Brain $(N=7)$} \\
\hline Ackerley et al. (88) & 12 & 18-35 & $12: 0$ & NR & Brush stroke & Palm & Y & $\mathrm{N}$ & fMRI \\
\hline Carey et al. (13)* & 5 & $33-80$ & $2: 3$ & $\mathrm{RH}$ & Texture grids & Fingertips & Y & Y (after scan) & PET \\
\hline Case et al. (89) & 26 & $\begin{array}{l}24.8(7) \\
19-43\end{array}$ & $11: 15$ & $\mathrm{RH}$ & Brush stroke & $\begin{array}{l}\text { Palm and } \\
\text { back of hand }\end{array}$ & Y & $\mathrm{N}$ & fMRI \\
\hline Hagen et al. $(61)^{\star}$ & 12 & $39(13)$ & $6: 6$ & $11 \mathrm{RH}, 1 \mathrm{LH}$ & Von Frey & Index finger & Y & $\mathrm{N}$ & PET \\
\hline Maldjian et al. (90) & 5 & $28-40$ & $4: 1$ & $\mathrm{RH}$ & Vibration & $\begin{array}{l}\text { Each finger } \\
\text { pad }\end{array}$ & NR & NR & fMRI \\
\hline Ozcan et al. (70)* & 12 & $22-35$ & $8: 4$ & $11 \mathrm{RH}, 1 \mathrm{LH}$ & Compressed air & Fingertips & $\mathrm{N}$ & $\mathrm{N}$ & $\mathrm{fMRI}$ \\
\hline Wacker et al. (91) & 13 & $22-35$ & $9: 4$ & 12RH, 1LH & Vibration & Index finger & Y & $\mathrm{N}$ & fMRI \\
\hline
\end{tabular}

*Studies contributing data to both RH and LH stimulation Whole-Brain analyses.

from the pooled image in an iterative process $(45,46)$. Contrast analyses permitted identification of regions of difference between groups while conjunction analyses quantify regions of overlap. To maximize accurate localization and interpretation, images created in GingerALE were also imported into the SPM Anatomy Toolbox (47-49) to permit localization of the ALE images with 3-dimensional probabilistic cytoarchitectonic mapping (50, 51). This regional cytoarchitectonic classification of ALE maps complements the GingerALE localization that uses peak MNI co-ordinates.

\section{RESULTS}

A total of $n=45$ studies were determined to be suitable for inclusion (see Table 1). Of the 45 studies, 29 were used to perform the $\mathrm{RH}$ whole-brain meta-analysis, seven were used for the LH whole-brain meta-analysis (three studies involved stimulation of both $\mathrm{LH}$ and $\mathrm{RH}$ independently), and 12 studies examined $\mathrm{RH}$ stimulation in a ROI analysis.

As can be seen in Table 1, for the $29 \mathrm{RH}$ whole-brain studies, a total of $n=375$ participants were included ( $n=173$ males, however $n=3$ studies did not report sex) aged 18-76 years. The RH ROI studies included $n=123$ participants $(n=63$ males) aged 18-66 years. The seven LH studies included $n=85$ participants ( $n=52$ males) aged $18-80$ years. The most common form of stimulation was vibration ( $n=12$ studies), followed by compressed air $(n=8)$, textures $(n=7)$, brush stroke $(n=7)$, Von Frey filaments $(n=4)$, and pressure $(n=4)$.

The RH whole-brain studies, RH ROI studies and the LH whole-brain studies were analyzed separately, as presented in Table 2 and Figure 2. For the RH whole-brain stimulation studies, the contralateral (left) primary and secondary somatosensory areas were significant, with a large cluster containing the parietal operculum (92), somatosensory (93), and motor (94) cortices. The ipsilateral (right) secondary somatosensory cortex, S2, was also significant, largely comprising the parietal operculum (92) and inferior parietal cortex $(95,96)$, in addition to a small cluster in the anterior cingulate. The $\mathrm{RH}$ ROI studies revealed visually smaller contralateral (left) clusters in the primary and secondary somatosensory regions, with a smaller ipsilateral (right) cluster within S2. The contralateral (left) clusters were separated into a large superior cluster containing the primary somatosensory (93) and motor (94) cortices, and a smaller inferior cluster containing primarily the parietal operculum (92). The ipsilateral (right) cluster contained similar areas to $\mathrm{RH}$ whole brain, namely the parietal operculum (92) and inferior parietal cortex $(95,96)$. With the small number of LH stimulation studies, only two clusters were significant in the contralateral (right) primary (containing somatosensory (93) and motor (94) cortices) and secondary somatosensory regions [primarily parietal operculum (92)] and primary auditory cortex (97) (Table 2).

Contrast analyses were then performed, as presented in Table 3 and Figure 3. When contrasted with the LH whole-brain studies, RH whole-brain studies revealed two clusters in the contralateral (left) primary and secondary somatosensory areas. The largest cluster contained primary somatosensory $(93,98)$ and motor (94) cortices, while the smaller cluster contained primarily the auditory cortex (97), insula (99), and parietal operculum (92). When contrasted with $\mathrm{RH}$ whole-brain studies, the LH whole-brain studies activated three small clusters in S1 quite similar to those found in the standalone LH whole-brain analysis, all containing primary somatosensory areas $(93,98)$. In our analysis of conjoined areas (i.e. areas of overlap) for $\mathrm{RH}$ and LH whole-brain studies, only one significant cluster 
TABLE 2 | Anatomical location, summary statistics and MNI co-ordinates of ALE identified areas for RH whole-brain, RH ROI and LH whole-brain studies (Extrema ALE value, FWE cluster corrected $p<0.05$, uncorrected $p<0.001$ ).

\begin{tabular}{|c|c|c|c|c|c|c|}
\hline SPM Anatomy Toolbox region location & MNI GingerALE peak location & Extrema value & Size & $x$ & $y$ & $z$ \\
\hline \multicolumn{7}{|c|}{ RH WHOLE-BRAIN STUDIES (30 CONTRASTS, 334 FOCI) } \\
\hline $\begin{array}{l}\text { Left parietal operculum (OP) Area OP3 } \\
\text { (VS), area OP4 (PV), and area OP1 (S2) }\end{array}$ & Left primary somatosensory area (S1); Insula (BA 13) & 0.061623 & 17,784 & -48 & -20 & 20 \\
\hline Left Area 1, Area 3b, and Area 4a & Left S1; postcentral gyrus (BA 2) & 0.035296 & & -54 & -20 & 48 \\
\hline Left Area 3b, Area 1, and Area 4a & Left S1; postcentral gyrus (BA2) & 0.028759 & & -44 & -26 & 58 \\
\hline Not assigned in probability maps & Left Insula (BA 13); claustrum & 0.021434 & & -38 & -12 & 4 \\
\hline Left Area OP4 (PV) & Left primary motor area (M1); insula (BA 13) & 0.016304 & & -44 & -8 & 10 \\
\hline Not assigned in probability maps & Left par opercularis (BA 44); insula (BA 13) & 0.015817 & & -40 & 4 & 10 \\
\hline $\begin{array}{l}\text { Right area OP1 (S2), area OP4 (PV), and } \\
\text { area TE } 1.0\end{array}$ & $\begin{array}{l}\text { Right supra marginal gyrus (SMG, BA 40); insula } \\
\text { (BA 13) }\end{array}$ & 0.039009 & 6,032 & 56 & -22 & 20 \\
\hline $\begin{array}{l}\text { Right area PFCm (inferior parietal lobe, } \\
\text { IPL), and Area OP1 (S2) }\end{array}$ & $\begin{array}{l}\text { Right superior temporal area (BA 22); insula } \\
\text { (BA 13) }\end{array}$ & 0.021011 & & 56 & -34 & 18 \\
\hline Right Area PFCm (IPL) and Area PF (IPL) & Right IPL, SMG (BA 40) & 0.017283 & & 56 & -38 & 28 \\
\hline $\begin{array}{l}\text { Right area PFop (IPL), area PFt (IPL), } \\
\text { and area } 3 b\end{array}$ & Right S1; postcentral gyrus (BA 2) & 0.015576 & & 60 & -20 & 32 \\
\hline Left area 33 & Left cingulate gyrus (BA 24, 32) & 0.022505 & 896 & -4 & 14 & 36 \\
\hline \multicolumn{7}{|l|}{ RH ROI STUDIES (12 CONTRASTS, 93 FOCI) } \\
\hline Left area 1 , area $4 a$ & Left S1; postcentral gyrus (BA 2) & 0.025885 & 6520 & -50 & -18 & 52 \\
\hline Left area $4 a$ and area $3 b$ & Left M1; postcentral gyrus (BA 3) & 0.017133 & & -40 & -28 & 60 \\
\hline Left area $4 a$ and $3 b$ & Left M1; postcentral gyrus (BA 3) & 0.015225 & & -42 & -22 & 58 \\
\hline $\begin{array}{l}\text { Left area OP1 (S2), area TE 1.0, and area } \\
\text { PFop (IPL) }\end{array}$ & Left postcentral gyrus, SMG, BA 40) & 0.014924 & 2,296 & -54 & -26 & 20 \\
\hline $\begin{array}{l}\text { Left area OP4 (PV), area OP3 (VS), and } \\
\text { area OP1 (S2) }\end{array}$ & Left S1; insula (BA 13) & 0.012088 & & -50 & -20 & 20 \\
\hline Area OP3 (VS) and Area OP4 (PV) & Left M1; Insula (BA 13) & 0.007544 & & -42 & -12 & 16 \\
\hline $\begin{array}{l}\text { Right Area OP1 (S2), Area PFcm (IPL), and } \\
\text { Area PFop (IPL) }\end{array}$ & Right IPL, SMG (BA 4) & 0.012395 & 1,840 & 54 & -26 & 24 \\
\hline Right area OP1 (S2) and area OP4 (PV) & Right SMG (BA 40); insula (BA 13) & 0.011046 & & 58 & -18 & 20 \\
\hline Right area OP4 (PV) & Right S1, postcentral gyrus (BA 43) & 0.007434 & & 60 & -8 & 14 \\
\hline \multicolumn{7}{|c|}{ LH WHOLE-BRAIN (7 CONTRASTS, 53 FOCI) } \\
\hline Right Area 1, Area 3b, and Area 4p & $\begin{array}{l}\text { Right primary somatosensory area (S1); postcentral } \\
\text { gyrus (BA2) }\end{array}$ & 0.013074 & 3,176 & 54 & -20 & 44 \\
\hline Right Area 3b, Area $4 p$, and Area $4 a$ & Right S1; IPL (BA40), postcentral gyrus & 0.010481 & & 40 & -34 & 60 \\
\hline Right Area 1 and Area 3b & Right S1; postcentral gyrus (BA 3) & 0.009132 & & 44 & -24 & 64 \\
\hline $\begin{array}{l}\text { Right Area 1, Area 3b, and Area PFt } \\
(I P L)\end{array}$ & Right S1; postcentral gyrus (BA 3) & 0.008071 & & 62 & -18 & 36 \\
\hline Right Area 4p, Area 3b, and Area 3 & Right S1; IPL (BA 40) & 0.007689 & & 36 & -34 & 52 \\
\hline Not assigned in probability maps & Right M1; precentral gyrus (BA 4) & 0.007664 & & 44 & -12 & 60 \\
\hline $\begin{array}{l}\text { Right area OP4 (PV), area OP1 and area } \\
\text { TE } 1.0\end{array}$ & $\begin{array}{l}\text { Right supramarginal gyrus (SMG: BA 40); Insula (BA } \\
\text { 13) }\end{array}$ & 0.016235 & 1,392 & 52 & -16 & 16 \\
\hline
\end{tabular}

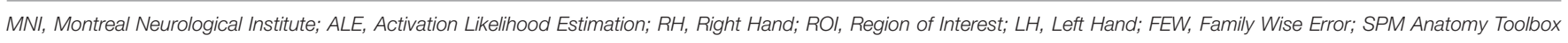

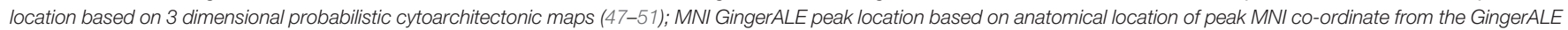

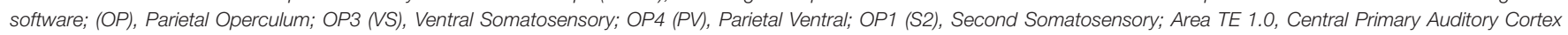

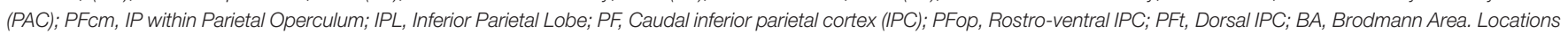
in italics refer to areas within the larger clusters (i.e. sub clusters identified).

was present, in the right secondary somatosensory region, primarily parietal operculum areas OP1, OP3 and OP4 (92). There were no significant differences in the contrast analysis between RH whole-brain studies and RH ROI studies. However, when the two groups were conjoined, significant common regions of activation were identified, with clusters revealed in the left primary (93) and secondary somatosensory areas (92), and the right secondary somatosensory area (92), including OP1.

\section{DISCUSSION}

In two important ways our ALE meta-analysis allowed us to examine the brain regions consistently activated during tactile 
stimulation of the hands in order to characterize functional somatosensory regions and networks, without the influence of motor function. Firstly, the meta-analysis allowed us to characterize and compare areas involved in right hand and left hand tactile stimulation studies separately. Secondly, it revealed the similarities and differences between functional activation studies that focus on specific brain regions (RH ROI studies) and what is actually occurring throughout the brain ( $\mathrm{RH}$ wholebrain studies). Unfortunately very few studies $(n=7)$ examined $\mathrm{LH}$ stimulation separate to the $\mathrm{RH}$ and without the influence of motor activity, making a statistical comparison between the hands difficult and exploratory.

For the RH whole-brain stimulation studies $(n=29)$ not only did we find two large clusters in the contralateral (left) primary (93) and secondary [specifically within parietal operculum areas OP1, OP3 and OP4 (92)] somatosensory cortices as expected, but activation was also revealed in the ipsilateral (right) secondary somatosensory region involving OP1 and OP4 (92) in addition to the anterior cingulate. Bilateral activation of secondary somatosensory S2 region, involving parietal operculum (92) to unilateral stimulation of the right hand is consistent with previous reports (100). From the few LH studies included, two small but significant clusters were revealed in the contralateral (right) S1 and S2. While each hand had significantly greater activation in the contralateral S1 and S2 in comparison to the other hand, the only significant area of overlap was in the right S2, specifically OP1, OP3 and OP4 (92). Lateralized differences have been reported for different sensory modalities, with right hemisphere being more spatially oriented toward the dorsal perceptual/sensory systems (101). Overlap in right S2 is consistent with hemispheric asymmetry involving righthemisphere-based bilateral representation of the body (101), right-sided asymmetry for tactile processing (102) and robust bilateral responses to unilateral stimulation in S2 (100). Due to the difference in numbers of studies included for each hand, this comparison is considered exploratory and highlights the need for more studies to examine LH tactile stimulation separately. Nevertheless, it is an interesting trend and could have significant implications for better understanding somatosensory function and dysfunction.

Activation in the contralateral S1, when using a tactile stimulus on the hand, is quite consistent with previous research $(13,14)$. The pattern of activation shown in the RH wholebrain studies is consistent with research showing contralateral S1 activation only, and studies that have shown bilateral activation in S2 regardless of the hand being stimulated $(16,17,103)$. It is surprising that bilateral S2 activation was not seen for the meta-analysis of LH studies also. However, this may have been attributable to the low number of studies stimulating the $\mathrm{LH}$ alone.

The role of S2 both contralateral and ipsilateral to the hand being stimulated is particularly interesting and may have important implications. The secondary somatosensory cortex of nonhuman primates is located on the parietal operculum, and the anatomical cytoarchitectonic maps of OP 1-4 of the human parietal operculum correlate with the functionally defined human somatosensory cortex (92), with OP 1 constituting the

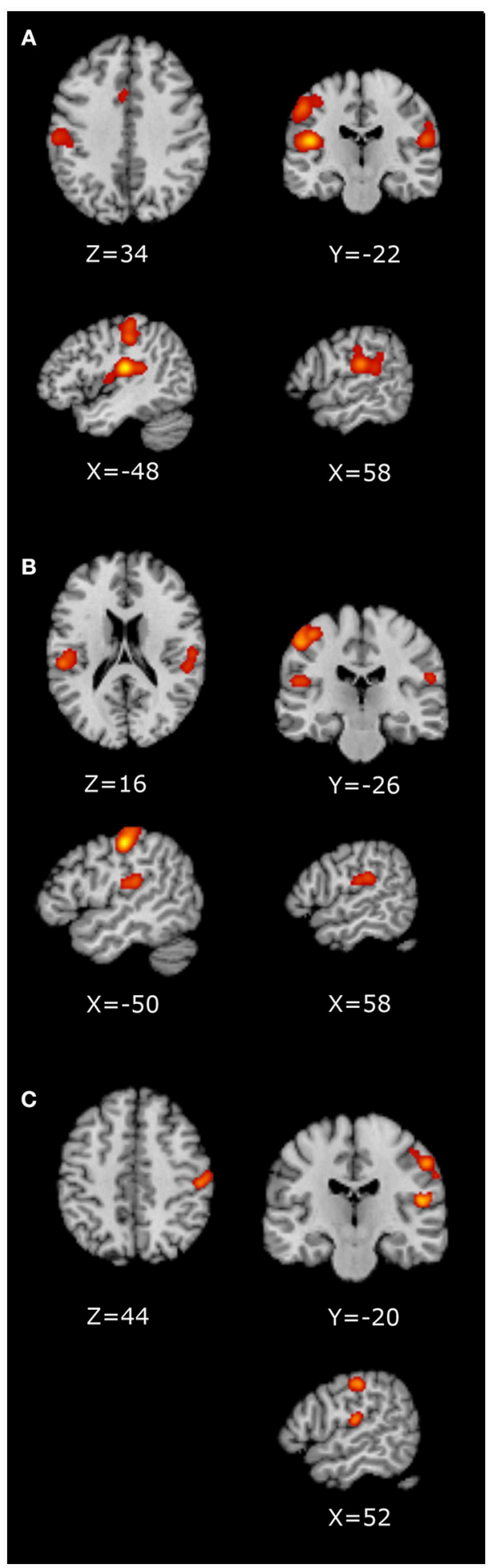

FIGURE 2 | ALE Images displayed in neurological convention. (A) RH whole-brain ALE; (B) RH ROI ALE; (C) LH whole-brain ALE.

putative human homologue of area S2 (92). Further, OP1 is closely connected with the parietal networks for higher order somatosensory processing, while OP 4 is more closely integrated 
TABLE 3 | Anatomical location, summary statistics and MNI coordinates of ALE identified areas for contrast analyses: RH Whole-Brain greater than LH Whole-Brain, LH Whole-Brain greater than $\mathrm{RH}$ Whole-Brain, $\mathrm{RH}$ Whole-Brain conjoined with LH Whole-Brain, and RH Whole-Brain conjoined with RH ROI studies $(p<0.01,10,000$ p-value permutations, $100 \mathrm{~mm}$ cluster threshold).

\begin{tabular}{|c|c|c|c|c|c|c|}
\hline SPM Anatomy Toolbox region location & MNI GingerALE peak location & Extrema value & Size & $\mathbf{x}$ & y & $\mathbf{z}$ \\
\hline \multicolumn{7}{|c|}{ RH WHOLE-BRAIN GREATER THAN LH WHOLE-BRAIN STUDIES } \\
\hline Left area $3 b$, area 2 and area $4 p$ & $\begin{array}{l}\text { Left Inferior parietal lobe (IPL), supramarginal gyrus } \\
\text { (SMG: BA 40) }\end{array}$ & 3.719017 & 4,168 & -45 & -28 & 44 \\
\hline Left area $4 p$, area $4 a$, and area $3 a$ & Left S1: postcentral gyrus (BA 2) & 3.540084 & & -49 & -25 & 50 \\
\hline Left area $4 a$ and area 1 & Left S1; postcentral gyrus (BA 2) & 3.352795 & & -52 & -19 & 53 \\
\hline Left area TE 1.0, area lg2, and area TE 1.1 & Left transverse temporal gyrus (BA 41) & 3.890592 & 3,488 & -39 & -22 & 17 \\
\hline $\begin{array}{l}\text { Left area TE 1.0, area TE 1.1, and area } \\
\text { OP1 (S2) }\end{array}$ & Left transverse temporal gyrus (BA 41) & 3.719017 & & -45 & -26 & 16 \\
\hline $\begin{array}{l}\text { Left area } \lg 2, \text { area TE } 1.2, \text { and area } \\
\text { TE } 1.0\end{array}$ & Left S1; insula (BA 13) & 3.352795 & & -44 & -18 & 12 \\
\hline Not assigned in probability maps & Left S1; postcentral gyrus (BA 2) & 3.011454 & & -44 & -20 & 28 \\
\hline \multicolumn{7}{|c|}{ LH WHOLE-BRAIN GREATER THAN RH WHOLE-BRAIN STUDIES } \\
\hline Right area $3 b$ and area 2 & Right S1: IPL (BA 40) & 2.597153 & 296 & 40 & -38 & 60 \\
\hline Right area $3 b$, area $4 p$, and area 2 & Right S1; IPL (BA 40) & 2.582808 & & 36 & -36 & 54 \\
\hline Not assigned in probability maps & Right S1; postcentral gyrus (BA 40) & 2.483769 & & 40 & -30 & 58 \\
\hline Right area 1 and area 3b & Right postcentral gyrus (BA 3) & 2.894304 & 288 & 45 & -26 & 58 \\
\hline Right area 1 and area 3b & Right postcentral gyrus (BA 3) & 2.911238 & 280 & 48 & -22 & 56 \\
\hline Right area 1 and area $3 b$ & Right postcentral gyrus (BA 3) & 2.847963 & & 52 & -20 & 52 \\
\hline Right area $3 b$ and area $4 a$ & Right postcentral gyrus (BA 2) & 2.575829 & & 48 & -18 & 54 \\
\hline Not assigned in probability maps & Right postcentral gyrus (BA 40) & 2.536396 & & 47 & -21 & 50 \\
\hline \multicolumn{7}{|c|}{ RH WHOLE-BRAIN STUDIES CONJOINED WITH LH WHOLE-BRAIN STUDIES } \\
\hline $\begin{array}{l}\text { Right area OP4 (PV), area OP1 (S2), and } \\
\text { area OP3 (V5) }\end{array}$ & Right SMG BA 40); insula (BA 13) & 0.016235 & 688 & 52 & -16 & 16 \\
\hline \multicolumn{7}{|c|}{ RH WHOLE-BRAIN CONJOINED WITH RH ROI STUDIES } \\
\hline Left area $3 b$, area $4 a$, and area 1 & Left S1; postcentral gyrus (BA 2) & 0.025433 & 4,400 & -50 & -18 & 50 \\
\hline Not assigned in probability maps & Left M1; postcentral gyrus (BA 3) & 0.017133 & & -40 & -28 & 60 \\
\hline Not assigned in probability maps & Left M1; postcentral gyrus (BA 3) & 0.015225 & & -42 & -22 & 58 \\
\hline $\begin{array}{l}\text { Left area OP1 (S2), area TE 1.0, and area } \\
\text { OP4 (PV) }\end{array}$ & Left postcentral gyrus, SMG (BA 40) & 0.014924 & 2,144 & -54 & -26 & 20 \\
\hline Not assigned in probability maps & Left S1; insula (BA 13) & 0.012088 & & -50 & -20 & 20 \\
\hline Not assigned in probability maps & Left M1; insula (BA 13) & 0.007544 & & -42 & -12 & 16 \\
\hline Right area OP1 (S2) and area OP4 (PV) & Right IPL, SMG (BA 40) & 0.012395 & 1,632 & 54 & -26 & 24 \\
\hline Not assigned in probability maps & Right insula (BA 13), SMG (BA 40) & 0.011046 & & 58 & -18 & 20 \\
\hline
\end{tabular}

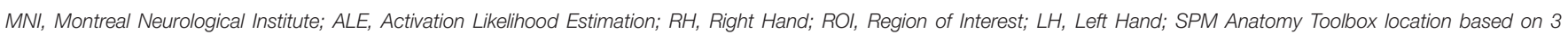




OP3 (VS), Ventral Somatosensory; BA, Brodmann Area. Locations in italics refer to areas within the larger clusters (i.e. sub clusters identified).

with areas responsible for basic sensorimotor processing and action control (104). Bilateral secondary somatosensory cortex, in particular, has demonstrated a role in complex integrative processes of stimulus elaboration and attention following stimulation of right hand (105). Tame, Braun (103) have demonstrated bilateral activation in both S1 and S2 regardless of which hand was stimulated, suggesting that these areas may be involved in integrating somatosensory input from both sides of the body. Some may attribute the involvement of ipsilateral S2 to a more cognitive role in sensory processing, and while it is important to consider the cognitive aspects of sensorimotor control, such as planning and strategy (106), bilateral S2 activation has been demonstrated in somatosensory studies regardless of the level of cognitive demand (18).
The involvement of S2 is particularly interesting in the context of aging, somatosensory dysfunction, and sensory rehabilitation. Age-related changes in activation have been seen, with decreased activation in S2 with tactile stimulation evident in elderly participants who are known to experience behavioral decline in somatosensory thresholds (54). The relationship of bilateral S2 with tactile sensation must also be considered in fields such as stroke research, where the location of the lesion has been demonstrated to impact both the type of somatosensory dysfunction (107), and also the ability to recover after stroke (108). Our finding of overlap in activation of right secondary somatosensory region for $\mathrm{RH}$ and $\mathrm{LH}$ tactile stimulation, may have particular relevance after stroke. For example, 


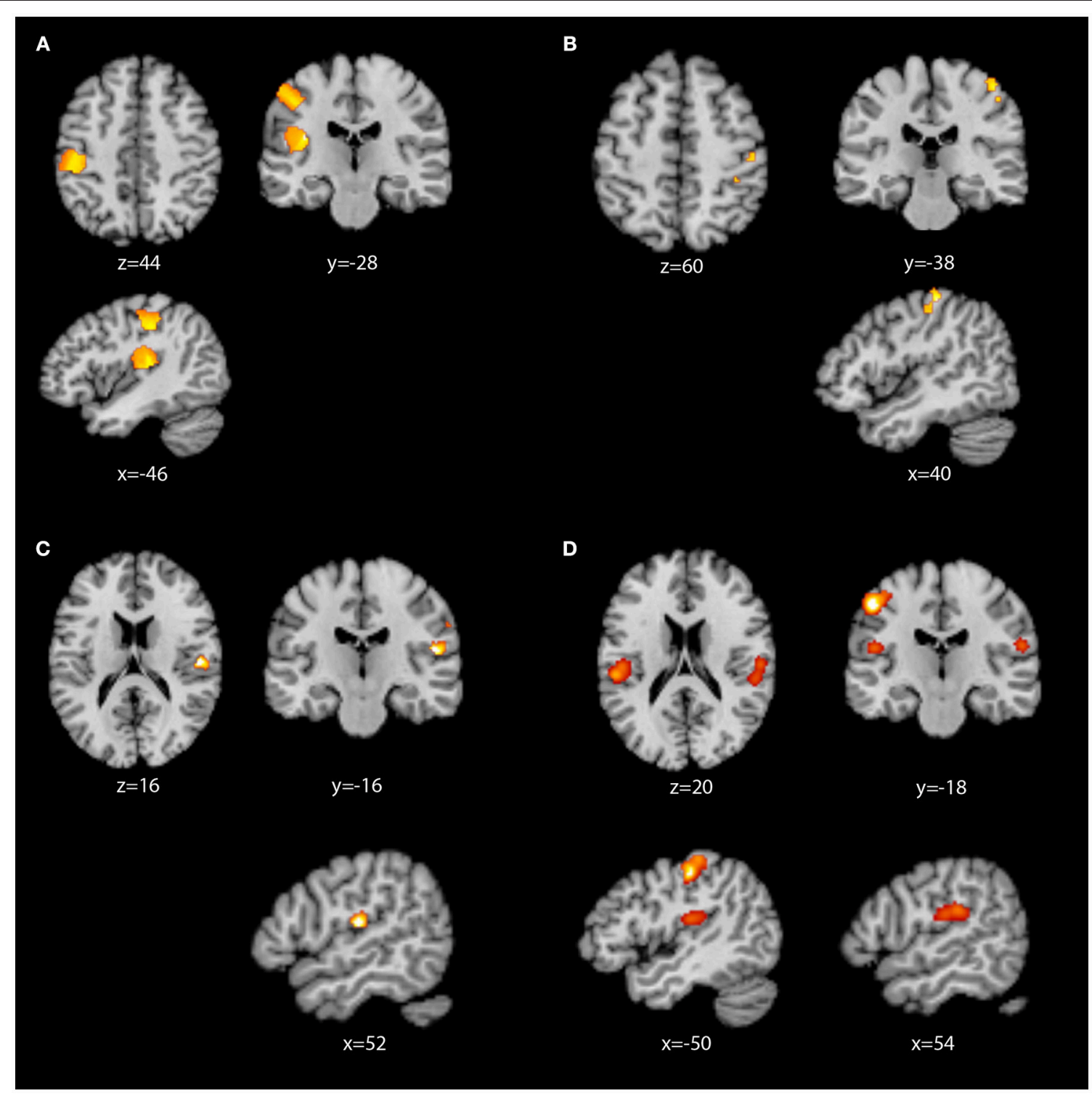

FIGURE 3 | ALE images displayed in neurological convention. (A) RH whole-brain activation greater than LH whole-brain activation; (B) LH whole-brain activation greater than $\mathrm{RH}$ whole-brain activation; (C) $\mathrm{RH}$ whole-brain conjoined with LH whole brain activation; (D) $\mathrm{RH}$ whole-brain conjoined with $\mathrm{RH} \mathrm{ROI}$ activation.

a stroke survivor with an infarct in the right hemisphere affecting S2 might not only experience the typically expected impairment of sensation in the contralateral hand (i.e., $\mathrm{LH}$ ), but also impairment in the ipsilateral right hand; as has been described clinically (2). Further, recent evidence of altered functional connectivity in stroke survivors with impaired touch sensation following left or right hemisphere lesions, highlighted increased laterality indices in ipsilateral (contralesional) S2 relative to healthy controls following lesion of either hemisphere (109). Further, functional connectivity research has demonstrated that an increase in connectivity from contralesional S2 to contralesional thalamus correlates with better somatosensory function 6-months post-stroke (110).
Evaluation of the RH ROI studies $(n=12)$ revealed that only contralateral (left) S1 and bilateral S2 were examined by studies which predefined the areas thought to be involved in somatosensory processing of the hand. In comparison, the $\mathrm{RH}$ whole-brain studies also revealed anterior cingulate activation, and much larger clusters were involved with tactile stimulation. This suggests that when researchers set out to examine the functional activation of a tactile stimulus, if they limit the focus to a-priori areas, this may not capture the entire neural functional process related to the sensation. Anterior cingulate activation may play a significant role in sensory processing. For example, pleasant human touch is represented in anterior cingulate cortex (111). In addition, while attention differentially modulates signal amplitudes in the human somatosensory cortex, 
at higher intensities activation is also seen in the anterior cingulate cortex, consistent with attention to tactile stimuli in the current studies (112). It has been suggested that Von Economo (spindle) neurons found in cingulate cortex (113), and linked with insula, may have a role as part of a salience network (114). Network analyses identify anterior cingulate as a hub region and common co-activation of anterior cingulate and insula support the interpretation of a saliency network devoted to the integration of information from internal and external sensory environments (115). Further, interhemispheric connections between bilateral thalami occur via the anterior cingulate (113) and healthy controls show interhemispheric functional connectivity between a number of regions associated with somatosensory processing, including anterior cingulate (107), highlighting the contribution of both hemispheres and the broader somatosensory system. Interestingly, cingulate cortex has also been implicated in rats sensory recovery after lesions (116).

Other areas identified in this meta-analysis included inferior parietal lobe, insula, supramarginal gyrus and temporal lobe. Inferior parietal lobe (IPL) of the right hemisphere was identified for both $\mathrm{RH}$ whole-brain and ROI analyses. The location included OP1 and OP4. IPL has been associated with multimodal sensory information integration $(117,118)$ and is reported to be part of the larger somatosensory network (119). The insula was also identified using the GingerALE peak maps, although this region was frequently assigned to the parietal operculum using the Anatomical toolbox. The insula has been identified as having a role in recognition, perception and learning in functional models of somatosensory processing (2). S2 is reciprocally connected with granular fields of the insula, reported to be devoted to somatic processing in monkeys (120). The close proximity of locations highlight the importance of the combined parietal opercular-insula region. Supramarginal gyrus is similarly located close to the parietal operculum/S2 region. The SMG is part of the somatosensory association cortex which has a role in interpretation of tactile sensory information as well as in perception of space and limbs location (121). Right SMG was found for RH whole-brain, RH ROI and LH whole-brain, and for the conjoined analyses. Right SMG is associated with spatial processing (121), consistent with tasks requiring localization of stimuli and/or involving spatial features of textures. Activation of left temporal gyrus, including auditory cortex and granular insula area 2, was greater in RH than LH whole-brain studies. Left temporal cortex has been linked with structural and semantic knowledge of body representation (122).

Each of the regions identified above have been implicated in stroke tactile impairment and recovery, potentially highlighting their broader importance. For example, change in functional connectivity from ipsilesional right S1 to right inferior parietal lobe was found in stroke survivors with impaired touch sensation compared to healthy controls (109). In addition, increased interhemispheric connectivity between the S2 region of interest and somatosensory association cortex (involving insula, parietal operculum and SMG) and temporal gyrus was found in healthy age-matched controls compared to stroke survivors with tactile deficits (109). Further, following tactile training, patients with lesions of sensory thalamus and/or internal capsule demonstrated activation in ipsiliesional insula, extending to the temporal pole, and supramarginal gyrus post-intervention (108). Interestingly, the regions identified have a role in the broader interpretation of tactile stimuli, including multi-modal integration, perception and learning, spatial processing and semantic knowledge and appear to be accessed as part of a wider somatosensory network.

There are limitations to this meta-analysis when examining the demographic information regarding the participants (see Table 1). Most of the LH studies (with the exception of one) included young participants (18-43 years). Aside from this, the cohorts were fairly well controlled, with the majority being $\mathrm{RH}$ dominant, and with tasks controlled for motor and other influences. Variable naming across studies can also contribute to confusion with interpretation. For example, terms such as secondary somatosensory cortex, secondary somatosensory region and secondary somatosensory area are often used interchangeably, although differences have been defined (10). To maximize accuracy and comparison across studies and broader literature in the field, we have reported on the MNI co-ordinates and peak location ALE results as well as the Anatomy Toolbox regional activation results.

The aim of this meta-analysis was to determine the convergence of foci reported from functional neuroimaging studies of touch sensation, separate to motor contributions and/or confounds. The findings advance our understanding of the separate, but potentially complementary, contributions of brain regions involved in processing touch sensation. Given the role of somatosensation and the somatosensory system in goaldirected actions of the upper limb and recovery after stroke, in depth knowledge of the role of key regions in the network is critical. The importance of bilateral S2 activation with right hand touch stimulation is highlighted, with a potential lateralization of activation in right S2 for right and left hand stimulation. This has implication for possible differences in unilateral vs. bilateral patterns of somatosensory impairment following right or left hemisphere lesion stroke. It may also identify a region with scope to contribute to recovery.

In conclusion, while research has established a role for S1 and S2 contralateral to the hand being stimulated $(13,14)$, this metaanalysis has demonstrated the need to also examine the bilateral activation in S2 with right hand stimulation in order to further delineate the role of this area in tactile processing. Additional studies examining $\mathrm{LH}$ tactile processing separate to the $\mathrm{RH}$ would be beneficial to further examine whether this same pattern of activation is seen. These two advances in understanding would in turn further research into somatosensory dysfunction and rehabilitation.

\section{AUTHOR CONTRIBUTIONS}

LC conceived the study. GL, LC, EL, and AB contributed to the design of the study. GL, SP, EL, and AB conducted the search and extraction of data. GL and PG conducted the meta-analysis. GL and LC interpreted the findings and drafted the manuscript. All authors critically reviewed and revised the manuscript for 
important intellectual content, provided approval for publication and agree to be accountable for all aspects of the work.

\section{FUNDING}

We acknowledge financial support for conduct of the research from the James S. McDonnell Foundation 21st

\section{REFERENCES}

1. Carey LM, Lamp G, Turville M. The state-of-the-science on somatosensory function and its impact on daily life in adults and older adults, and following stroke: a scoping review. OTJR (2016) 36:275-415. doi: $10.1177 / 1539449216643941$

2. Dijkerman HC, de Haan EH. Somatosensory processing subserving perception and action: dissociations, interactions, and integration. Behav Brain Sci. (2007) 30:224-30. doi: 10.1017/S0140525X07001641

3. Galazky I, Schutze H, Noesselt T, Hopf JM, Heinze HJ, Schoenfeld MA. Attention to somatosensory events is directly linked to the preparation for action. J Neurol Sci. (2009) 279:93-8. doi: 10.1016/j.jns.2008.12.006

4. Blennerhassett JM, Carey LM, Matyas TA. Clinical measures of handgrip limitation relate to impaired pinch grip force control after stroke. J Hand Ther. (2008) 21:245-53. doi: 10.1197/j.jht.2007.10.021

5. Tremblay F, Wong K, Sanderson R, Cote L. Tactile spatial acuity in elderly persons: assessment with gratings domes and relationship with manual dexterity. Somatosens Mot Res. (2003) 20:127-32. doi: 10.1080/0899022031000105154

6. Blennerhassett JM, Matyas TA, Carey LM. Impaired discrimination of surface friction contributes to pinch grip deficit after stroke. Neurorehabil Neural Repair (2007) 21:263-72. doi: 10.1177/1545968306295560

7. Turville M, Carey L, Matyas T, Blennerhassett J. Change in functional arm use is associated with somatosensory skills following sensory retraining post-stroke. Am J Occup Ther. (2017) 71:24950. doi: 10.5014/ajot.2017. 024950

8. Rehme AK, Eickhoff SB, Rottschy C, Fink GR, Grefkes C. Activation likelihood estimation meta-analysis of motor-related neural activity after stroke. Neuroimage (2012) 59:2771-82. doi: 10.1016/j.neuroimage.2011.10.023

9. Carey LM. Somatosensory loss after stroke. Crit Rev Phys Rehabil Med. (1995) 7:51-91. doi: 10.1615/CritRevPhysRehabilMed.v7.i1.40

10. Eickhoff SB, Grefkes C, Zilles K, Fink GR. The somatotopic organization of cytoarchitectonic areas on the human parietal operculum. Cereb Cortex. (2007) 17:1800-11. doi: 10.1093/cercor/bhl090

11. Head H, Holmes G. Sensory disturbances from cerebral lesions. Brain (1911) 34:102-254. doi: 10.1093/brain/34.2-3.102

12. Merabet LB, Pascual-Leone A. Neural reorganization following sensory loss: the opportunity of change. Nat Rev Neurosci. (2010) 11:44-52. doi: $10.1038 / \mathrm{nrn} 2758$

13. Carey LM, Abbott DF, Egan GF, Donnan GA. Reproducible activation in BA2, 1 and $3 \mathrm{~b}$ associated with texture discrimination in healthy volunteers over time. Neuroimage (2008) 39:40-51. doi: 10.1016/j.neuroimage.2007.08.026

14. Sanchez-Panchuelo R-M, Besle J, Mougin O, Gowland P, Bowtell R, Schluppeck D, et al. Regional structural differences across functionally parcellated Brodmann areas of human primary somatosensory cortex. Neuroimage (2014) 93:221-30. doi: 10.1016/j.neuroimage.2013.03.044

15. van Ede F, de Lange FP, Maris E. Anticipation increases tactile stimulus processing in the ipsilateral primary somatosensory cortex. Cereb Cortex (2014) 24:2562-71. doi: 10.1093/cercor/bht111

16. Boakye M, Huckins SC, Szeverenyi NM, Taskey BI, Hodge CJ. Functional magnetic resonance imaging of somatosensory cortex activity produced by electrical stimulation of the median nerve or tactile stimulation of the index finger. J Neurosurg. (2000) 93:774-83. doi: 10.3171/jns.2000.93. 5.0774
Century Science Initiative in Cognitive Rehabilitation Collaborative Award (\#220020413). We also acknowledge support from the National Health and Medical Research Council of Australia (NHMRC) Project grant (\#1022694), Career Development Award (\#307905) Centre of Research Excellence (\#1077898) and Partnership grant (\#1134495).

17. Lee SD, Jung Y, Chung YA, Lee W. Neural substrates in secondary somatosnesory area for the perception of different tactile sensations. Int $J$ Imaging Syst Technol. (2016) 26:85-91. doi: 10.1002/ima.22160

18. Albanese M-C, Duerden EG, Bohotin V, Rainville P, Duncan GH. Differential effects of cognitive demand on human cortical activation associated with vibrotactile stimulation. J Neurophysiol. (2009) 102:1623-31. doi: 10.1152/jn.91295.2008

19. Khoshnejad M, Piche M, Saleh S, Duncan G, Rainville P. Serial processing in primary and secondary somatosensory cortex: A DCM analysis of human fMRI data in response to innocuous and noxious electrical stimulation. Neurosci Lett. (2014) 577:83-8. doi: 10.1016/j.neulet.2014.06.013

20. Eickhoff SB, Nichols TE, Laird AR, Hoffstaeder F, Amunts K, Fox PT, et al. Behavior, sensitivity, and power of activation likelihood estimation characterized by massive empirical simulation. Neuroimage (2016) 137:7085. doi: 10.1016/j.neuroimage.2016.04.072

21. Burton H, Sinclair R, McLaren D. Cortical activity to vibrotactile stimulation: an fMRI study in blind and sighted individuals. Hum Brain Mapp. (2004) 23:210-28. doi: 10.1002/hbm.20064

22. Bak N, Glenthoi BY, Rostrup E, Larsson HB, Oranje B. Source localization of sensory gating: a combined EEG and fMRI study in healthy volunteers. Neuroimage (2011) 54:2711-8. doi: 10.1016/j.neuroimage.2010.11.039

23. Bodegard A, Geyer S, Naito E, Zilles K, Roland PE. Somatosensory areas in man activated by moving stimuli: cytoarchitectonic mapping and PET. NeuroReport (2000) 11:187-91. doi: 10.1097/00001756-200001170-00037

24. Ghazni NF, Cahill CM, Stroman PW. Tactile sensory and pain networks in the human spinal cord and brain stem mapped by means of functional MR imaging. Am J Neuroradiol. (2010) 31:661-7. doi: 10.3174/ajnr.A1909

25. Boecker H, KhorramSefat D, Kleinschmidt A, Merboldt KD, Hanicke W, Requardt $\mathrm{M}$, et al. High-resolution functional magnetic resonance imaging of cortical activation during tactile exploration. Hum Brain Mapp. (1995) 3:236-44. doi: 10.1002/hbm.460030307

26. Disbrow E, Roberts T, Krubitzer L. Somatotopic organization of cortical fields in the lateral sulcus of Homosapiens: evidence for SII and PV. J Compar Neurol. (2000) 418:1-21. doi: 10.1002/(SICI)1096-9861(20000228)418:1<1::AID-CNE1>3.0.CO;2-P

27. Gelnar PA, Krauss BR, Sheehe PR, Szeverenyl NM, Apkarian AV. A comparative fMRI study of cortical representations for thermal painful, vibrotactile, and motor performance tasks. Neuroimage (1999) 10:460-82. doi: 10.1006/nimg.1999.0482

28. Haller S, Chapuis D, Gassert R, Burdet E, Klarhofer M. Supplementary motor area and anterior intraparietal area integrate fine-graded timing and force control during precision grip. Euro J Neurosci. (2009) 30:2401-6. doi: 10.1111/j.1460-9568.2009.07003.x

29. Jackson SR, Parkinson A, Pears SL, Nam SH. Effects of motor intention on the perception of somatosensory events: a behavioural and functional magnetic resonance imaging study. Q J Exp Psychol. (2011) 64:839-54. doi: $10.1080 / 17470218.2010 .529580$

30. Moher D, Liberati A, Tetzlaff J, Altman DG, The PRISMA Group. Preferred reporting items for systematic reviews and metaanalyses: the PRISMA statement. PLoS Med. (2009) 6:e1000097. doi: 10.1371/journal.pmed.1000097

31. Fox PT, Laird AR, Fox SP, Fox PM, Uecker AM, Crank M, et al. BrainMap taxonomy of experimental design: description and evaluation. Hum Brain Mapp. (2005) 25:185-98. doi: 10.1002/hbm.20141

32. Fox PT, Lancaster JL. Mapping context and content: the BrainMap model. Nature Rev Neurosci. (2002) 3:319-21. doi: 10.1038/nrn789 
33. Laird AR, Lancaster JL, Fox PT. BrainMap: the social evolution of a functional neuroimaging database. Neuroinformatics (2005) 3:65-78. doi: 10.1385/NI:3:1:065

34. Eickhoff SB, Bzdok D, Laird AR, Kurth F, Fox PT. Activation likelihood estimation revisited. Neuroimage (2012) 59:2349-61. doi: 10.1016/j.neuroimage.2011.09.017

35. Eickhoff SB, Laird AR, Grefkes C, Wang LE, Zilles K, Fox PT. Coordinatebased activation likelihood estimation meta-analysis of neuroimaging data: a random-effects approach based on empirical estimates of spatial uncertainty. Hum Brain Mapp. (2009) 30:2907-26. doi: 10.1002/hbm.20718

36. Turkeltaub PE, Eickhoff SB, Laird AR, Fox M, Wiener M, Fox P. Minimizing within-experiment and within-group effects in activation likelihood estimation meta-analyses. Hum Brain Mapp. (2012) 33:1-13. doi: 10.1002/hbm.21186

37. Eickhoff SB, Laird AR, Fox PM, Lancaster JL, Fox PT. Implementation errors in the GingerALE Software: description and recommendations. Hum Brain Mapp. (2017) 38:7-11. doi: 10.1002/hbm.23342

38. Collins DL, Neelin P, Peters TM, Evans AC. Automatic intersubject registration of MR volumetric data in standardized talairach space. J Comp Assist Tomogr. (1994) 18:192-205. doi: 10.1097/00004728-199403000-00005

39. Evans AC, Collins DL, Mills SR, Brown ED, Kelly RL, Peters TM. 3D statistical neuroanatomical models from 305 MRI volumes. In: IEEE Conference Record on Nuclear Science Symposium and Medical Imaging Conference (San Francisco, CA) (1993).

40. Talairach J, Tournoux P. Co-planar Stereotaxic Atlas of the Human Brain. New York, NY: Thieme (1988).

41. Laird AR, Robinson C, McMillan KM, Tordesillas-Gutierrez D, Moran ST, Gonzales SM, et al. Comparison of the disparity between Talairach and MNI coordinates in functional neuroimaging data: validation of the Lancaster transform. Neuroimage (2010) 51:677-83. doi: 10.1016/j.neuroimage.2010.02.048

42. Lancaster JL, Tordesillas-Gutierrez D, Martinez M, Salinas F, Evans A, Zilles K, et al. Bias between MNI and Talairach coordinates analyzed using the ICBM-152 brain template. Hum Brain Mapp. (2007) 28:1194-205. doi: 10.1002/hbm.20345

43. Genovese CR, Lazar NA, Nichols T. Thresholding of statistical maps in functional neuroimaging using the false discovery rate. Neuroimage (2002) 15:870-8. doi: 10.1006/nimg.2001.1037

44. Laird AR, Fox PM, Price CJ, Glahn DC, Uecker AM, Lancaster JL, et al. ALE meta-analysis: controlling the false discovery rate and peforming statistical contrasts. Hum Brain Mapp. (2005) 25:155-64. doi: 10.1002/hbm.20136

45. Eickhoff SB, Bzdok D, Laird AR, Roski C, Caspers S, Zilles K, et al. Co-activation patterns distinguish cortical modules, their connectivity and functional differentiation. Neuroimage (2011) 57:938-49. doi: 10.1016/j.neuroimage.2011.05.021

46. Fox PT, Laird AR, Eickhoff SB, Lancaster JL, Fox M, Uecker AM, et al. User Manual for GingerALE 2.3. San Antonio, TX: UT Health Science Center San Antonio; Research Imaging Institute (2013).

47. Eickhoff SB, Stephan KE, Mohlberg H, Grefkes C, Fink GR, Amunts K, et al. A new SPM toolbox for combining probabilistic cytoarchitectonic maps and functional imaging data. Neuroimage (2005) 25:1325-35. doi: 10.1016/j.neuroimage.2004.12.034

48. Eickhoff SB, Heim S, Zilles K, Amunts K. Testing anatomically specified hypotheses in functional imaging using cytoarchitectonic maps. Neuroimage (2006) 32:570-82. doi: 10.1016/j.neuroimage.2006.04.204

49. Eickhoff SB, Paus T, Caspers S, Grosbras M-H, Evans AC, Zilles $\mathrm{K}$, et al. Assignment of functional activations to probabilistic cytoarchitectonic areas revisited. Neuroimage (2007) 36:511-21. doi: 10.1016/j.neuroimage.2007.03.060

50. Zilles K, Amunts K. Centenary of Brodmann's map- conception and fate. Nature Rev Neurosci. (2010) 11:139-45. doi: 10.1038/nrn2776

51. Amunts K, Schleicher A, Zilles K. Cytoarchitecture of the cerebral cortex - more than localization. Neuroimage (2007) 37:1061-5. doi: 10.1016/j.neuroimage.2007.02.037

52. Borstad A, Schmalbrock P, Choi S, Nichols-Larsen DS. Neural correlates supporting sensory discrimination after left hemisphere stroke. Brain Res. (2012) 1460:78-87. doi: 10.1016/j.brainres.2012.03.060
53. Bjornsdotter M, Gordon I, Pelphrey KA, Olausson H, Kaiser MD Development of brain mechanisms for processing affective touch. Front Behav Neurosci. (2014) 8:24. doi: 10.3389/fnbeh.2014.00024

54. Brodoehl S, Klingner C, Stieglitz K, Witte OW. Age-related changes in the somatosensory processing of tactile stimulation- an fMRI study. Behav Brain Res. (2013) 238:259-64. doi: 10.1016/j.bbr.2012.10.038

55. Brodoehl S, Klingner CM, Witte OW. Eye closure enhances dark night perceptions. Sci Rep. (2015) 5: 10515. doi: 10.1038/srep10515

56. Brodoehl S, Klingner C, Witte OW. Age-dependent modulation of the somatosensory network upon eye closure. Behav Brain Res. (2016) 298:52-6. doi: 10.1016/j.bbr.2015.10.035

57. Burton H, MacLeod AMK, Videen TO, Raichle ME. Multiple foci in parietal and frontal cortex activated by rubbing embossed grating patterns across fingerpads: a Positron emission tomography study in humans. Cereb Cortex (1997) 7:3-7. doi: 10.1093/cercor/7.1.3

58. Chung YG, Han SW, Kim HS, Chung SC, Park JY, Wallraven C, et al. Intra- and inter-hemispheric effective connectivity in the human somatosensory cortex during pressure stimulation. BMC Neurosci. (2014) 15:43. doi: 10.1186/1471-2202-15-43

59. Chung YG, Han SW, Kim HS, Chung SC, Park JY, Wallraven C, et al. Adaptation of cortical activity to sustained pressure stimulation on the fingertip. BMC Neurosci. (2015) 16:71. doi: 10.1186/s12868-015-0207-x

60. Godde B, Diamond ME, Braun C. Feeling for space or for time: taskdependent modulation of the cortical representation of identical vibrotactile stimuli. Neurosci Lett. (2010) 480:143-7. doi: 10.1016/j.neulet.2010.06.027

61. Hagen MC, Zald DH, Thornton TA, Pardo JV. Somatosensory processing in the human inferior prefrontal cortex. J Neurophysiol. (2002) 88:1400-6. doi: 10.1152/jn.2002.88.3.1400

62. Hlushchuk Y, Hari R. Transient suppression of ipsilateral primary somatosensory cortex during tactile finger stimulation. J Neurosci. (2006) 26:5819-24. doi: 10.1523/JNEUROSCI.5536-05.2006

63. Kavounoudias A, Roll JP, Anton JL, Nazarian B, Roth M, Roll R. Proprio-tactile integration for kinesthetic perception: an fMRI study. Neuropsychologia (2008) 46:567-75. doi: 10.1016/j.neuropsychologia.2007.10.002

64. Kitada R, Kochiyama T, Hashimoto T, Naito E, Matsumura M. Moving tactile stimuli of fingers are integrated in the intraparietal and inferior parietal cortices. Neuroreport (2003) 14:719-24. doi: 10.1097/00001756-200304150-00012

65. Kitada R, Hashimoto T, Kochiyama T, Kito T, Okada T, Matsumura M, et al. Tactile estimation of the roughness of gratings yields a graded response in the human brain: an fMRI study. Neuroimage (2005) 25:90-100. doi: 10.1016/j.neuroimage.2004.11.026

66. Kwon HG, Jang SH, Lee MY. Effects of visual information regarding tactile stimulation on the somatosensory cortical activation: a functional MRI study. Neural Regenerat Res. (2017) 12:1119-23. doi: 10.4103/1673-5374.211191

67. Malinen S, Schurmann M, Hlushchuk Y, Forss N, Hari R. Improved differentiation of tactile activation in human secondary somatosensory cortex and thalamus using cardiac-triggered fMRI. Exp Brain Res. (2006) 174:297-303. doi: 10.1007/s00221-006-0465-z

68. McGlone F, Olausson H, Boyle JA, Jones-Gotman M, Dancer C, Guest S, et al. Touching and feeling: diffrences in pleasant touch processing between glabrous and hairy skin in humans. Eur J Neurosci. (2012) 35:1782-8. doi: 10.1111/j.1460-9568.2012.08092.x

69. Nebel MB, Folger S, Tommerdahl M, Hollins M, McGlone F, Essick G. Temporomandibular disorder modifies cortical response to tactile stimulation. J Pain (2010) 11:1083-94. doi: 10.1016/j.jpain.2010.02.021

70. Ozcan M, Baumgartner U, Vucurevic G, Stoeter P, Treede RD. Spatial resolution of fMRI in the human parasylvian cortex: comparison of somatosensory and auditory activation. Neuroimage (2005) 25:877-87. doi: 10.1016/j.neuroimage.2004.11.037

71. Planetta PJ, Servos P. The postcentral gyrus shows sustained fMRI activation during the tactile motion aftereffect. Exp Brain Res. (2012) 216:535-44. doi: 10.1007/s00221-011-2957-8

72. Rolls ET, O’Doherty J, Kringelbach ML, Francis S, Bowtell R, McGlone F. Representations of pleasant and painful touch in the human 
orbitofrontal and cingulate cortices. Cerebr Cortex (2003) 13:308-17. doi: $10.1093 /$ cercor/13.3.308

73. Ruben J, Schwiemann J, Deucherr, M, Meyer R, Krause T, Curio G, et al. Somatotopic organization of human secondary somatosensory cortex. Cereb Cortex (2001) 11:463-73. doi: 10.1093/cercor/11.5.463

74. Schurmann M, Caetano G, Hlushchuk Y, Jousmaki V, Hari R. Touch activates human auditory cortex. Neuroimage (2006) 30:1325-31. doi: 10.1016/j.neuroimage.2005.11.020

75. Summers IR, Francis ST, Bowtell RW, McGlone FP, Clemence M. A functional-magnetic-resonance-imaging investigation of cortical activation from moving vibrotactile stimuli on the fingertip. J Acoust Soc Am. (2009) 125:1033-9. doi: 10.1121/1.3056399

76. Yoo SC, Freeman DK, McCarthy JJ, Jolesz FA. Neural substrates of tactile imagery: a functional MRI study. Neuroreport (2003) 14:581-5. doi: 10.1097/00001756-200303240-00011

77. Young JP, Herath P, Eickhoff S, Choi J, Grefkes C, Zilles K, et al. Somatotopy and attentional modulation of the human parietal and opercular regions. $J$ Neurosci. (2004) 24:5391-9. doi: 10.1523/JNEUROSCI.4030-03.2004

78. Blankenburg F, Ruben J, Meyer R, Schwiemann J, Villringer A. Evidence for a rostral-to-caudal somatotopic organization in human primary somatosensory cortex with mirror-reversal in areas $3 \mathrm{~b}$ and 1 . Cereb Cortex (2003) 13:987-93. doi: 10.1093/cercor/13.9.987

79. Blatow M, Nennig E, Durst A, Sartor K, Stippich C. fMRI reflects functional connectivity of human somatosensory cortex. Neuroimage (2007) 37:927-36. doi: 10.1016/j.neuroimage.2007.05.038

80. Blatow M, Reinhardt J, Riffel K, Nennig E, Wengenroth M, Stippich C. Clinical functional MRI of sensorimotor cortex using passive motor and sensory stimulation at 3 tesla. J Magn Reson Imaging (2011) 34:429-37. doi: 10.1002/jmri.22629

81. Burton H, Sinclair RJ, McLaren DG. Cortical network for vibrotactile attention: a fMRI study. Hum Brain Mapp. (2008) 29:207-21. doi: $10.1002 / \mathrm{hbm} .20384$

82. Deuchert M, Ruben J, Schweimann J, Meyer R, Thees S, Krause T, et al. Eventrelated fMRI of the somatosensory system using eletrical finger stimulation. Neuroreport (2002) 13:365-9. doi: 10.1097/00001756-200203040-00023

83. Dresel C, Parzinger A, Rimpau C, Zimmer C, Ceballos-Baumann AO, Haslinger B. A new device for tactile stimulation during fMRI. Neuroimage (2008) 39:1094-103. doi: 10.1016/j.neuroimage.2007.09.033

84. Huang RS, Sereno MI. Dodecapus: an MR-compatible system for somatosensory stimulation. Neuroimage (2007) 34:1060-73. doi: 10.1016/j.neuroimage.2006.10.024

85. Kobayashi M, Takeda K, Kaminaga T, Shimizu T, Iwata M. Neural consequences of somatosensory extinction - an fMRI study. J Neurol. (2005) 252:1353-8. doi: 10.1007/s00415-005-0865-1

86. Martuzzi R, van der Zwagg W, Farthouat J, Gruetter R, Blanke O. Human finger somatotopy in areas $3 \mathrm{~b}, 1$, and 2: a $7 \mathrm{~T}$ fMRI study using a natural stimulus. Hum Brain Mappi. (2014) 35:213-26. doi: 10.1002/hbm.22172

87. Nelson AJ, Chen R. Digit somatotopy within cortical areas of the postcentral gyrus in humans. Cereb Cortex (2008) 18:2341-51. doi: 10.1093/cercor/bhm257

88. Ackerley R, Hassan, E, Curran A, Wessberg J, Olausson H, McGlone F. An fMRI study on cortical responses during active self-touch and passive touch from others. Front Behav Neurosci. (2012) 6:51. doi: 10.3389/fnbeh.2012.00051

89. Case LK, Laubacher CM, Olausson H, Wang B, Spagnolo PA, Bushnell MC. Encoding of touch intensity but not pleasantness in human primary somatosensory cortex. J Neurosci. (2016) 36:5850-60. doi: 10.1523/JNEUROSCI.1130-15.2016

90. Maldjian JA, Gottschalk A, Patel RS, Detre JA, Alsop DC. The sensory somatotopic map of the human hand demonstrated at 4 Tesla. Neuroimage (1999) 10:55-62. doi: 10.1006/nimg.1999.0448

91. Wacker E, Spitzer B, Lutzkendorf R, Bernarding J, Blankenburg F. Tactile motion and pattern processing assessed with high-field fMRI. PLOS ONE (2011) 6:e24860. doi: 10.1371/journal.pone.0024860

92. Eickhoff SB, Amunts K, Mohlberg H, Zilles K. The human parietal operculum. II. Stereotaxic maps and correlation with functional imaging results. Cereb Cortex (2006) 16:268-79. doi: 10.1093/cercor/bhi106
93. Geyer S, Schormann T, Mohlberg H, Zilles K. Areas 3a, 3b and 1 of human primary somatosensory cortex. 2. Spatial normalization to standard anatomical space. Neuroimage (2000) 11:684-96. doi: $10.1006 /$ nimg. 2000.0548

94. Geyer S, Ledberg A, Schleicher A, Kinomura S, Schormann T, Burgel U, et al. Two different areas within the primary cortex of man. Nature (1996) 382:805-7. doi: $10.1038 / 382805 \mathrm{a} 0$

95. Scheperjans F, Eickhoff SB, Homke L, Mohlberg H, Hermann K, Amunts K, et al. Probabilistic maps, morphometry, and variability of cytoarchitectonic areas in the human superior parietal cortex. Cereb Cortex (2008) 18:2141-57. doi: $10.1093 /$ cercor/bhm 241

96. Scheperjans F, Hermann K, Eickhoff SB, Amunts K, Schleicher A, Zilles $\mathrm{K}$. Observer-independent cytoarchitectonic mapping of the human superior parietal cortex. Cereb Cortex (2008) 18:846-67. doi: 10.1093/cercor/bhm116

97. Morosan P, Rademacher J, Schleicher A, Amunts K, Schormann T, Zilles K. Human primary auditory cortex: cytoarchitectonic subdivisions and mapping into a spatial reference system. Neuroimage (2001) 13:684-701. doi: 10.1006/nimg.2000.0715

98. Grefkes C, Geyer S, Schormann T, Roland P, Zilles K. Human somatosensory area 2: Observer-independent cytoarchitectonic mapping, interindividual variability and population map. Neuroimage (2001) 14:617-31. doi: 10.1006/nimg.2001.0858

99. Kurth F, Eickhoff SB, Schleicher A, Hoemke L, Zilles K, Amunts K. Cytoarchitecture and probabilistic maps of the human posterior insular cortex. Cereb Cortex (2010) 20:1448-61. doi: 10.1093/cercor/bhp208

100. Eickhoff SB, Grefkes C, Fink GR, Zilles K. Functional lateralization of face, hand, and trunk representation in anatomically defined human somatosensory areas. Cereb Cortex (2008) 18:2820-30. doi: 10.1093/cercor/bhn039

101. Vallar G. A hemispheric asymmetry in somatosensory processing. Behav Brain Sci. (2007) 30:223-4. doi: 10.1017/S0140525X0700163X

102. Harada T, Saito DN, Kashikura KI, Sato T, Yonekura Y, Honda M, et al. Asymmetrical neural substrates of tactile discrimination in humans: a functional magnetic resonance imaging study. J Neurosci. (2004) 24:7524-30. doi: 10.1523/JNEUROSCI.1395-04.2004

103. Tame L, Braun C, Lignau A, Schwarzbach J, Demarchi G, Hegner YL, et al. The contribution of primary and secondary somatosensory cortices to the representation of body parts and body sides: an fMRI adaptation study. $J$ Cogn Neurosci. (2012) 24:2306-20. doi: 10.1162/jocn_a_00272

104. Eickhoff SB, Jbabdi S, Caspers S, Laird AR, Fox PT, Zilles K, et al. Anatomical and functional connectivity of cytoarchitectonic areas within the human parietal operculum. J Neurosci. (2010) 30:6409-21. doi: 10.1523/JNEUROSCI.5664-09.2010

105. Chen TL, Babiloni C, Ferretti A, Perrucci MG, Romani GL, Rossini PM, et al. Effects of somatosensory stimulation and attention on human somatosensory cortex: an fMRI study. Neuroimage (2010) 53:181-8. doi: 10.1016/j.neuroimage.2010.06.023

106. Borich MR, Brodie SM, Gray WA, Ionta S, Boyd LA. Understanding the role of the primary somatosensory cortex: opportunities for rehabilitation. Neuropsychologia (2015) 79:246-55. doi: 10.1016/j.neuropsychologia.2015.07.007

107. Ben-Shabat E, Matyas T, Pell GS, Brodtmann A, Carey LM. The right supramarginal gyrus is improtant for proprioception in healthy and strokeaffected participants: a functional MRI study. Front Neurol. (2015) 6:248. doi: 10.3389/fneur.2015.00248

108. Carey LM, Abbott DF, Lamp G, Puce A, Seitz RJ, Donnan GA. Same intervention-different reorganization: the impact of lesion location on training-facilitated somatosensory recovery after stroke. Neurorehabil Neural Repair. (2016) 30:988-1000. doi: 10.1177/1545968316653836

109. Goodin P, Lamp G, Vidyasagar R, McArdle D, Seitz RJ, Carey LM. Altered functional connectivity differs in stroke survivors with impaired touch sensation following left and right hemisphere lesions. Neuroimage Clin. (2018) 18:342-55. doi: 10.1016/j.nicl.2018.02.012

110. Bannister LC, Crewther SG, Gavrilescu M, Carey LM. Improvement in touch sensation after stroke is associated with resting functional connectivity changes. Front Neurol. (2015) 6:165. doi: 10.3389/fneur.2015. 00165 
111. Lindgren L, Westling G, Brulin C, Lehtipalo S, Andersson M, Nyberg L. Pleasant human touch is represented in pregenual anterior cingulate cortex. Neuroimage (2012) 59:3427-32. doi: 10.1016/j.neuroimage.2011.11.013

112. Arthurs OJ, Johansen-Berg H, Matthews PM, Boniface SJ. Attention differentially modulates the coupling of PMRI BOLD and evoked potential signal amplitudes in the human somatosensory cortex. Exper Brain Res. (2004) 157:269-74. doi: 10.1007/s00221-003-1827-4

113. Rao's V, Bentivoglio M. Crosstalk between the two sides of the thalamus through the reticular nucleus: a retrograde and anterograde tracing study in the rat. J Compar Neurol. (1993) 332:145-54. doi: 10.1002/cne.903320202

114. Stevens FL, Hurley RA, Taber KH. Anterior cingulate cortex: unique role in cognition and emotion. J Neuropsych Clin Neurosci. (2011) 23:121-5. doi: 10.1176/jnp.23.2.jnp121

115. Torta DM, Costa T, Duca S, Fox PT, Cauda F. Parcellation of the cingulate cortex at rest and during tasks: a meta-analytic clustering and experimental study. Front Hum Neurosci. (2013) 7:275. doi: 10.3389/fnhum.2013.00275

116. Abo M, Chen Z, Lail L-J, Reese T, Bjelke B. Functional recovery after brain lesion- contralateral neuromodulation: an fMRI study. NeuroReport (2001) 12:1543-7. doi: 10.1097/00001756-200105250-00048

117. Beauchamp MS, Pasalar S, Ro T. Neural substrates of reliability-weighted visual-tactile multisensory integration. Front Syst Neurosci. (2010) 4:25. doi: $10.3389 /$ fnsys.2010.00025

118. Gentile G, Petkova VI, Ehrsson HH. Integration of visual and tactile signals from the hand in the human brain: an FMRI study. J Neurophysiol. (2011) 105:910-22. doi: 10.1152/jn.00840.2010
119. Avanzini P, Abdollahi RO, Sartori I, Caruana F, Pelliccia V, Casaceli $\mathrm{G}$, et al. Four-dimensional maps of the human somatosensory system. Proc Natl Acad Sci USA. (2016) 113:E1936-43. doi: 10.1073/pnas.16018 89113

120. Schneider RJ, Friedman DP, Mishkin M. A modality-specific somatosensory area within the insula of the rhesus monkey. Brain Res. (1993) 621:116-20. doi: 10.1016/0006-8993(93)90305-7

121. Naito E, Roland PE, Grefkes C, Choi HJ, Eickhoff S, Geyer S, et al. Dominance of the right hemisphere and role of area 2 in human kinesthesia. J. Neurophysiol. (2005) 93:1020-34. doi: 10.1152/jn.00637.2004

122. Schwoebel J, Coslett HB. Evidence for multiple, distinct representations of the human body. J Cogn Neurosci. (2005) 17:543-53. doi: 10.1162/0898929053467587

Conflict of Interest Statement: The authors declare that the research was conducted in the absence of any commercial or financial relationships that could be construed as a potential conflict of interest.

Copyright $\odot 2019$ Lamp, Goodin, Palmer, Low, Barutchu and Carey. This is an open-access article distributed under the terms of the Creative Commons Attribution License (CC BY). The use, distribution or reproduction in other forums is permitted, provided the original author(s) and the copyright owner(s) are credited and that the original publication in this journal is cited, in accordance with accepted academic practice. No use, distribution or reproduction is permitted which does not comply with these terms. 